\title{
Esophageal ECG: The Challenge of Electrode Design
}

\author{
Thomas Niederhauser, Andreas Haeberlin, Rolf Vogel \\ ARTORG Cardiovascular Engineering \\ University of Bern \\ Bern, Switzerland \\ e-mail: thomas.niederhauser@artorg.unibe.ch
}

\author{
Thanks Marisa, Josef Goette, Marcel Jacomet \\ Microlab - Human Centered Engineering \\ Bern University of Applied Sciences \\ Biel, Switzerland
}

\begin{abstract}
Two commercially available electrode catheters are examined for their suitability in esophageal long-term ECG recordings. Both, electrical sensing characteristics as well as clinical acceptance were investigated in a clinical study including inpatients with cardiovascular diseases. In total, 31 esophageal ECG were obtained in 36 patients. Results showed that esophageal electrodes were well tolerated by the patients. Hemispherical electrodes with higher diameter required more insertion attempts and were associated with increased failure rates as compared to cylindrical electrodes. In contrast, the higher surface area of hemispherical electrodes resulted in significantly higher signal-to-noise ratio. Contact impedance was equal for both electrode types, but esophageal electrodes had lower impedance if compared with skin electrodes.
\end{abstract}

Keywords - esophageal electrodes, esophageal ECG, noise, contact impedance, insertion attempts.

\section{INTRODUCTION}

Heart rhythm disorders (arrhythmias) can be associated with fatal outcomes such as cerebral apoplexy, syncope or sudden cardiac death. The surface electrocardiogram (ECG) using skin electrodes attached to the chest wall and the limbs is the clinical gold standard to detect the presence and characterize the type of arrhythmia. However, many patients suffer only from short-lasting, rarely occurring episodes, and, therefore, require long-term ECG recordings in order to make the diagnosis and to define the therapeutic strategy. The probability to detect such paroxysmal arrhythmias increases with the recording duration and signal quality [1], [2], [3].

The sensing electrode is the most crucial link in the ECG signal acquisition chain, and its performance exerts high impact on the overall signal quality, and, in turn, clinical performance of the technique. Conventional silver/silver-chloride $(\mathrm{Ag} / \mathrm{AgCl})$ skin electrodes are clinically well established, but come with major disadvantages. They require conductive gels and adhesive films, both may dry out during long-term recording, thus electrical contact gets lost. Furthermore, they can cause skin irritation or even provoke allergic reactions. As an alternative, dry electrodes have been investigated. While these dry electrodes may prevent skin irritation, they are associated with higher skin-electrode impedance, higher susceptibility to motion artifact [4], and higher noise level [5]. Thus, signal acquisition with consistency and high quality remains a challenge, and highly depends on how and where electrodes are attached to the body [6], [7]. Overall, the lead-off rate is high for all types of surface electrodes used in ambulatory setting
[8], and increases with prolonged recording times requiring electrode replacement [1].

As compared to the body surface, the esophagus guarantees optimal mechanical, electrical and biological characteristics for the electrode placement in order to capture the ECG, because the collapsed esophagus generates contact pressure between electrode and underlying tissue. The esophageal mucus with its intrinsic clearance acts as conductive electrolyte. Due to the close proximity to the heart [9], esophageal electrodes sense both atrial as well as ventricular electrical signals with high quality [10].

Although the clinical feasibility of esophageal long-term recording has already been shown in 1978 [11], [12], digital signal processing and machine interpretation of esophageal ECG (eECG) is lacking. This is because the signals suffer from high baseline wander, which is associated with electrode motion due to respiration, esophageal peristalsis, and cardiac motion [13]. The frequency spectra of these noise sources clearly overlap with the ECG signal bandwidth making appropriate digital filtering difficult and, in turn, leading to higher wave detection errors [14], [15].

In order to improve the technique of transesophageal atrial stimulation (TAS), the effects of body position, inter-electrode spacing, and electrode surface area were assessed [16]. None of these parameters had significant influence to pacing thresholds. However, sensing properties of esophageal electrodes have never been studied. Therefore, the main goal of this study was to investigate different electrode designs for future use in longterm esophageal ECG recording, focusing on signal capturing capability and contact impedance.

Furthermore, long-term intubation of the esophagus is clinically well accepted and tolerated in several fields, e.g. nasogastric feeding. Nevertheless, those types of catheters are different to esophageal probes regarding stiffness and tube material. Here we present an evaluation of esophageal catheters using different diameters and electrode designs with respect to usability for clinicians and acceptance of patients.

\section{METHODS}

\section{A. Study Population}

For this study, 36 patients of the inpatient clinic of the Department of Cardiology, Bern University Hospital were recruited. The patients, 6 females and 30 males, aged 28 to 81 years (mean $63 \pm 13$ years), were hospitalized for coronary

This work was funded by the Commission for Technology and Innovation CTI, Switzerland, the Bern University Hospital and the Swiss Heart Foundation. 
artery disease, heart failure, or arrhythmias. Patients were included into the study irrespective of known arrhythmias as well as the presence or absence of pathologic findings in the surface ECG. The height and weight of the patient ranged from 1.57 to $1.90 \mathrm{~m}$ (mean $1.73 \pm 0.08 \mathrm{~m}$ ), and from 60 to $136 \mathrm{~kg}$ (mean $88 \pm 16 \mathrm{~kg}$ ), respectively. The study was approved by the ethical committee of the Canton Bern and informed consent was obtained from each patient.

\section{B. Esophageal Electrodes}

Two types of hexapolar esophageal electrodes from FIAB $\mathrm{SpA}$, Italy, were used in this study. Catheter 1 (ESOFLEX $6 \mathrm{~S}^{\circledR}$ ) was made of polyurethane with a body diameter of $7 \mathrm{~F}$ $(2.3 \mathrm{~mm})$. The ring electrodes had a hemispherical shape $(11 \mathrm{~F}$, $3.6 \mathrm{~mm}$ ) and were made of stainless steel. The catheter consisted of 1 distal pole (labeled 1) and 5 proximal poles (labeled 2-6), each with a surface area of $0.57 \mathrm{~cm}^{2}$. The interdistance between the electrodes was $15 \mathrm{~mm}$ from edge to edge, except between electrode 1 and 2, where the distance was 20 mm. Catheter 2 (ESOSOFT $6 \mathrm{~S}^{\circledR}$ ) was made of a 9F (3 mm) silicone tube. Unlike catheter 1 , the electrode rings had a cylindrical shape and the same diameter as the body. Each stainless steel ring had a surface area of $0.47 \mathrm{~cm}^{2}$. The electrodes had the same spatial segmentation and were labeled in an analogous manner as catheter 1 .

\section{Measurement Setup}

Each patient underwent measurements of (a) bipolar eECG signals and (b) impedance of the electrode-body system using different esophageal leads (see Table 1). eECG signals were amplified and digitized by a multichannel biosignal monitor (BioRadio ${ }^{\circledR} 150$, CleveMed Inc, USA) and transmitted over RF link to a personal computer. The 8 differential amplifiers had an input $(-3 \mathrm{~dB})$ bandwidth of $0.5-250 \mathrm{~Hz}$. No additional filters were applied. eECG data were sampled at $960 \mathrm{~Hz}$ using an input range from $\pm 3 \mathrm{mV}$ to $\pm 12 \mathrm{mV}$. The raw eECG data were analyzed offline using MATLAB ${ }^{\circledR}$. Before and after eECG recordings, the impedance was measured using an USB DAQ system (NI USB ${ }^{\circledR} 6215$, National Instruments Corp., USA) by stimulating the system with a stationary sinusoidal signal $(10 \mathrm{~Hz}, 200 \mathrm{mV}$ amplitude). Both, voltage and current (over a shunt resistor of $1000 \Omega$ ) were differentially measured with sampling rate of $25 \mathrm{kHz}$. Amplitude and phase of the impedance were calculated using custom-made, LabVIEW $^{\circledR}$ based software that achieved 4 values per second. Raw impedance data were subsequently analyzed offline using MATLAB.

\section{Measurement Procedure}

The esophageal probe was lubricated with oxybuprocaine gel and inserted into the esophagus via the nasal route in semisupine position. All patients were in non-sedated state. Two bipolar leads were configured, eLead 1 between electrode 2 and 6 and eLead 2 between electrode either 3 or 4 (randomly assigned) and 5. Electrode 1 served as ground (see Table 1). The electrode position was carefully adjusted until $\mathrm{P}$ waves with maximal amplitude were obtained in eLead 2. Having found the optimal position, the catheter was fixed at nasal wings and impedance measurements, each lead once during a period of $1 \mathrm{~min}$, were performed (pre). Thereafter, the eECG of both leads was synchronously measured during a time window of max 2 hours. Afterwards, the impedance measurement was repeated (post). Finally, the catheter was removed and the patient was asked about discomfort that was evaluated by a scoring system (0-30) including 10 clinical parameters such as gag reflex, nasal pain, sneezing, coughing etc. rated by 0 to 3 points.

\section{E. Signal and Statistical Analysis}

Pre and post impedance was calculated by the mean of the samples recorded during $1 \mathrm{~min}(\bar{Z})$, each with the amplitude and phase. The Mann-Whitney-U test was used to compare means of the different configurations.

The signal-to-noise ratio (SNR) of the eECG was estimated by calculating the signal band energy and out-of-band noise energy (1), (2). The signal was defined as the energy in the signal band between $f_{B W}=2 \mathrm{~Hz}$ and $f_{S t}=40 \mathrm{~Hz} ; 2 \mathrm{~Hz}$ is the frequency which is assumed to be the upper limit of the baseline wander [17], [13]; and $40 \mathrm{~Hz}$ is the standard $-3 \mathrm{~dB}$ frequency bandwidth for ambulatory electrocardiographic systems [18]. The remaining spectrum was considered as noise. Assuming a zero mean wide-sense stationary random signal (WSS signal) $x[\mathrm{n}][19]$, the variance of $x[\mathrm{n}]$ denoted by $\sigma_{x}^{2}$ is

$$
\sigma_{x}^{2}=E\left\{|x[\mathrm{n}]|^{2}\right\}=r_{x}[0]=\frac{1}{2 \pi} \int_{-\pi}^{\pi} P_{x}\left(e^{j \omega}\right) d \omega,
$$

where $r_{x}[\mathrm{n}]$ is the autocorrelation of $x[\mathrm{n}]$ and $P_{x}\left(e^{j \omega}\right)$ is its discrete-time Fourier transform. The total power of the WSS signal $x[\mathrm{n}]$ is therefore proportional to the area under the power spectral density curve. Using (1), a one-sided estimation $\hat{P}_{x}\left(e^{j \omega}\right)$ of $P_{x}\left(e^{j \omega}\right)$, and the sampling frequency $f_{s}$, the SNR becomes

$$
\begin{gathered}
\text { SNR }=\frac{\sigma_{\text {Signal }}^{2}}{\sigma_{\text {Noise }}^{2}}= \\
\frac{\int_{f_{B W}}^{f_{S t}} \hat{P}_{x}\left(e^{j 2 \pi f / f_{s}}\right) d f}{\int_{0}^{f_{B W}} \hat{P}_{x}\left(e^{j 2 \pi f / f_{s}}\right) d f+\int_{f_{S t}}^{f_{s} / 2} \hat{P}_{x}\left(e^{j 2 \pi f / f_{s}}\right) d f}
\end{gathered}
$$

The estimation $\hat{P}_{x}\left(e^{j \omega}\right)$ was calculated for 1-min segments of eECG signals (seg) with Welch's Method [19], using Hamming windows of length $\mathrm{L}=20 \cdot f_{s}$ with $50 \%$ overlap. Besides the mean ( $\overline{\mathrm{SNR}})$, the number of segments with SNR $\left(\mathrm{SNR}_{\text {seg }}\right)$ greater than a predefined threshold (thr) divided by the total number of segments $N$ was calculated:

$$
\begin{gathered}
N_{\text {seg }}(\text { thr })=\frac{1}{\mathrm{~N}} \sum_{\mathrm{i}=1}^{\mathrm{N}} c\left(\operatorname{seg}_{i}\right), \\
c\left(\operatorname{seg}_{i}\right)= \begin{cases}1, & \mathrm{SNR}_{\mathrm{seg}_{i}}>0 \\
0, & \text { else }\end{cases}
\end{gathered}
$$

SNR data of the different configurations are compared, again using the Mann-Whitney-U test. 


\section{RESULTS}

\section{A. Electrical Properties}

We obtained eECG signals in 31 out of 35 patients $(86 \%)$ with mean $\pm \mathrm{SD}$ recording time of $114 \pm 34$ minutes. Fig. 1 illustrates two characteristic eECG traces from two different leads of ESOFLEX probe. Both leads suffered from baseline wander due to respiration and cardiac motion: SNR for eLead 1 and eLead 2 were $1.92 \mathrm{~dB}$ and $-3.318 \mathrm{~dB}$, respectively. Nevertheless, $\mathrm{P}$ and $\mathrm{R}$ waves are clearly visible in both leads with $\mathrm{P}$ waves having greater amplitudes in eLead 2 despite lower SNR. Fig. 2 shows an example of two esophageal signals recorded with the ESOSOFT probe, whereby esophageal peristalsis caused high baseline wander disturbance. Although both leads yielded low SNR $(<-16 \mathrm{~dB}), \mathrm{P}$ and $\mathrm{R}$ waves are again not difficult to determine.

Table 1 summarizes impedance and SNR values of the two probes with the different leads. The impedance of the various leads and probes did not differ significantly. Furthermore, no significant impedance changes resulted during the eECG recording. However, mean signal-to-noise ratio as well as the number of segments above a certain threshold varied strongly with the electrode type being used.

Fig. 2 shows the box plot of the SNR analysis (top). Obviously, ESOFLEX electrodes exhibited higher SNR as compared to ESOSOFT electrodes $(\mathrm{p}=0.009)$. The difference was significant using a threshold of $-6 \mathrm{~dB}(\mathrm{p}=0.046)$ and lost significance considering only the segments with SNR $>0 \mathrm{~dB}(\mathrm{p}$ $=0.09$ ). Different leads of the same probe type did not affect the mean SNR; however eLead 1 generated the lower interquartile range. The variance of SNR in each group of eECG was high, e.g. ranging from $-20.5 \mathrm{~dB}$ to $12.4 \mathrm{~dB}$ with ESOFLEX 6S, eLead 1. Compared to baseline wander energy, the eECG energy was lower in $71 \%$ to $93 \%$ of the segments.

\section{B. Clinical Evaluation}

Probe insertion failed in 2 patients with nasal septum deviation, in 2 patients with strong gag reflex and in 1 patient with sneezing fits. Discomfort was highest during probe insertion (mean 2.9 points), decreased immediately after insertion (mean 1.2 points, $\mathrm{p}<0.00001$ ) and further declined
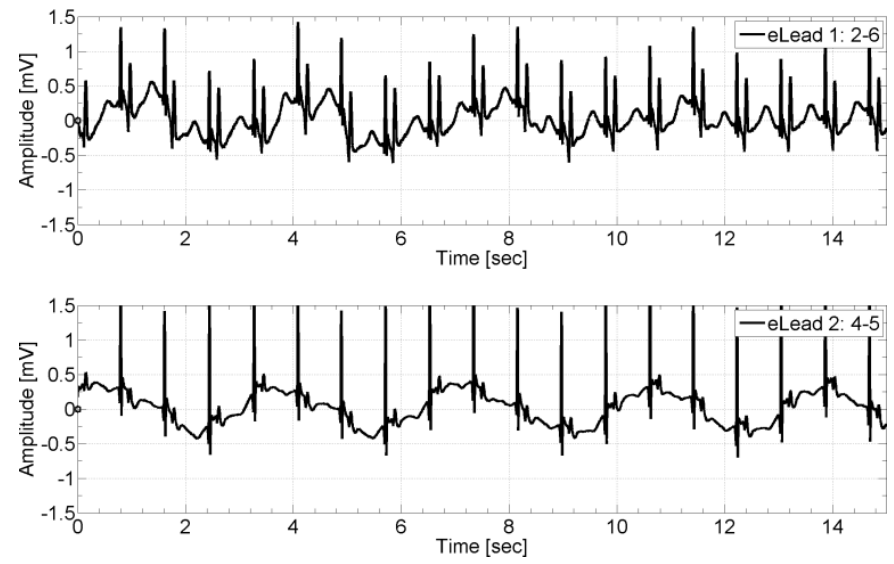

Figure 1. Characteristic eECG signals using ESOFLEX 6S; top: eLead 1 (2-6), bottom: eLead 2 (4-5).
TABLE I. IMPEDANCE AND SNR OF TWO ESOPHAGEAL PROBES.

\begin{tabular}{|c|l|l|l|l|l|}
\hline \multirow{2}{*}{$\begin{array}{c}\text { Esophageal } \\
\text { Probe / Lead }\end{array}$} & \multicolumn{2}{|c|}{$|\bar{Z}|(\mathbf{\Omega})$} & \multicolumn{1}{c|}{$\overline{\text { SNR (dB) }}$} & $\begin{array}{c}N_{\text {seg }} \\
(\mathbf{0} \text { dB) }\end{array}$ & $\begin{array}{c}N_{\text {seg }} \\
(-6 \text { dB) }\end{array}$ \\
\cline { 2 - 6 } & $\widetilde{Z}$, pre & $\widetilde{Z}$, post & $\widetilde{S N R}$, iqr & $\widetilde{\boldsymbol{N}}_{\text {seg }}$ & $\widetilde{\boldsymbol{N}}_{\text {seg }}$ \\
\hline ESOFLEX 6S & 4291 & 5527 & $-6.89,16.48$ & $26 \%$ & $43 \%$ \\
\hline eLead 1: 2-6 & 4055 & 4451 & $-7.15,9.01$ & $29 \%$ & $46 \%$ \\
\hline eLead 2: 3-5 & $\mathrm{n} / \mathrm{a}$ & $\mathrm{n} / \mathrm{a}$ & $\mathrm{n} / \mathrm{a}$ & $\mathrm{n} / \mathrm{a}$ & $\mathrm{n} / \mathrm{a}$ \\
\hline eLead 2: 4-5 & 5193 & 6556 & $-6.64,16.35$ & $21 \%$ & $38 \%$ \\
\hline ESOSOFT 6S & 6758 & 5248 & $-16.30,12.31$ & $9 \%$ & $21 \%$ \\
\hline eLead 1: 2-6 & 6299 & 5628 & $-15.68,10.73$ & $12 \%$ & $22 \%$ \\
\hline eLead 2: 3-5 & 6336 & 4203 & $-19.04,22.30$ & $8 \%$ & $22 \%$ \\
\hline eLead 2: 4-5 & 7173 & 6061 & $-20.25,12.61$ & $7 \%$ & $14 \%$ \\
\hline
\end{tabular}

a. Most data were not normal distributed, $\tilde{x}=$ median, iqr $=$ interquartile range b. Data included all leads of this probe type

until the probe was removed (mean 1.0 point, $\mathrm{p}=0.014$, vs. after insertion). The type of esophageal probe had no influence on discomfort. However, the number of insertion attempts was larger in patients receiving an ESOFLEX as compared to patients with an ESOSOFT probe $(\mathrm{p}=0.021)$, see Fig. 2 (bottom). We obtained 11 eECG recordings with the ESOFLEX (22 attempts, success rate 50\%) and 20 with the ESOSOFT (22 attempts, success rate 91\%). Patients, whereby the ESOFLEX insertion failed, received alternatively an ESOSOFT probe.

\section{DISCUSSION}

Impedance measurements showed no difference between different leads and electrode types. Larger surface area $(0.57$ $\mathrm{cm}^{2}$ vs. $0.47 \mathrm{~cm}^{2}$ ) was not associated with better and more stable contact properties. It is not the gross geometry, but rather the material and the microscopic surface area (roughening, sputtering etc.) of the electrode that can significantly reduce the contact impedance [20].

Our data showed that esophageal electrodes own better contact properties with the underlying tissue than surface electrodes, whose skin contact impedance is greater than $10 \mathrm{k} \Omega$
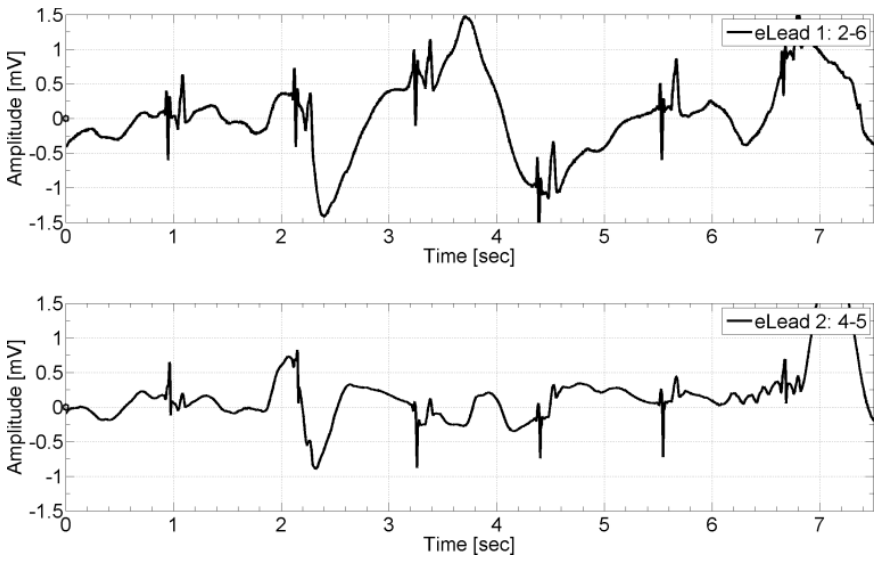

Figure 2. eECG signals disturbed by esophageal peristalsis (ESOSOFT 6S); top: eLead 1 (2-6), bottom: eLead 2 (4-5). 

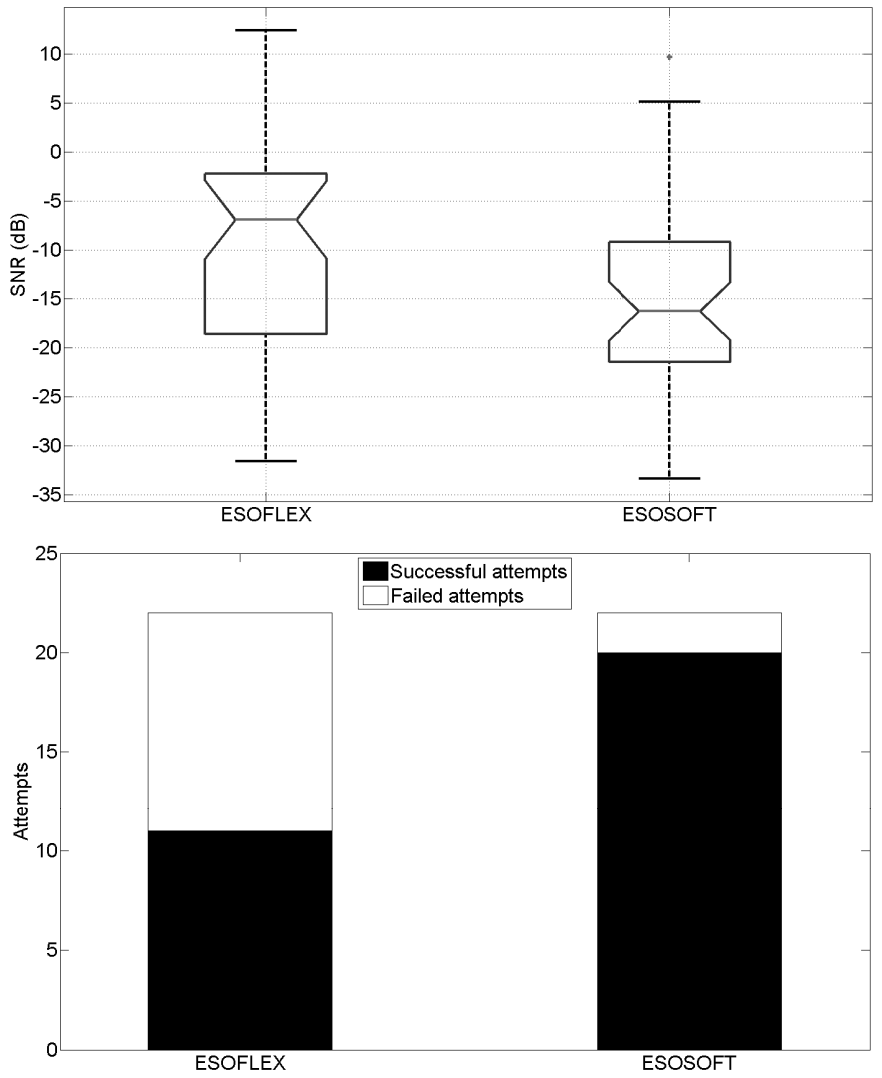

Figure 3. Comparison of ESOFLEX (left) and ESOSOFT probe (right); top: Signal-to-noise ratio, bottom: Insertion attempts.

at $10 \mathrm{~Hz}$ [21]. The reasons for these better properties are the esophageal mucus with its steady clearance acting as a conducting electrolyte, and the continuous pressure of the collapsed esophagus.

In contrast to the impedance, the SNR data varied significantly between the two probe types, regardless of the leads being used. Hemispheric as compared to cylindrical electrodes increased the SNR by $9.5 \mathrm{~dB}$ in average. Baseline wander as the main source of noise is directly related to electrode motion and thus is correlated to impedance variation [13]. Therefore, the hemispherical electrodes captured more signal energy in the required bandwidth. This leads to the assumption that electrodes with an increased surface area, while maintaining their inter-electrode distance, yield eECG signals with higher amplitudes. It should be noted that eECG signal quality also depends on patient's anatomy, spatial relationship between esophagus and heart, and electrode location. Consistently, the inter-patient variability explains the high variance of SNR measurements. Using the hemispherical electrode, $3 / 4$ of all eECG segments contained more noise than signal energy. However, $\mathrm{R}$ and $\mathrm{P}$ waves are still clearly visible in most of the segments, and might be well detected by appropriate signal processing.

The type of esophageal probe, i.e. catheter material and electrode design, had an impact on catheter insertion and the eECG signal quality. The catheter with the higher body diameter (ESOSOFT vs. ESOFLEX, $9 \mathrm{~F}$ vs. $7 \mathrm{~F}$ ), but smaller electrode diameter ( $9 \mathrm{~F}$ vs. $11 \mathrm{~F}$ ) was easier to handle. It is obvious that insertion success is highly defined by the maximal tube diameter. However, the probe type did not affect the patient's discomfort, neither during insertion nor with progressing recording time. Overall, patient discomfort, which was described as a foreign body feeling, was little, and all patients with successful probe insertion completed the study.

\section{CONCLUSION}

Esophageal ECG (eECG) is well tolerated by the patients and easy to perform by clinicians. Smaller esophageal electrode diameters $(<9 \mathrm{~F})$ are favorable from a clinical point of view but impair signal quality. Signal quality can be improved by using hemispherical electrodes with larger surface areas. Although the signal energy is mostly concentrated in the low frequency range (baseline wander noise), clinically relevant eECG waves are clearly visible. Considering the better contact properties and higher atrial sensitivity, the esophageal approach is a reliable and promising alternative to conventional long-term ECG recording systems using surface skin electrodes.

Further investigation has to be done in electrode design as well as appropriate analog signal amplification and digital signal processing. The eECG signals containing high baseline wander require advanced filtering techniques, which have to be explored.

\section{ACKNOWLEDGMENT}

The authors would like to thank all the clinical staff from the Department of Cardiology, Bern University Hospital involved in this study for their assistance.

\section{BIBLIOGRAPHY}

[1] Hans Kottkamp et al., "Time Courses and Quantitative Analysis of Atrial Fibrillation Episode Number and Duration After Circular Plus Linear Left Atrial Lesions," J. Am. Coll. Cardiol., vol. 44, no. 4, pp. 869-77, August 2004.

[2] Rypko Beukema et al., "Monitoring of atrial fibrillation burden after surgical ablation: relevancy of end-point criteria after radiofrequency ablation treatment of patients with lone atrial fibrillation," Interact. CardioVasc. Thorac. Surg., vol. 9, pp. 956-959, September 2009.

[3] Raoul Stahrenberg et al., "Enhanced Detection of Paroxysmal Atrial Fibrillation by Early and Prolonged Continuous Holter Monitoring in Patients With Cerebral Ischemia Presenting in Sinus Rhythm," Stroke, vol. 41, pp. 00-00, October 2010, in press.

[4] Timo Vuorela, Ville Pekka Seppä, Jukka Vanhala, and Jari Hyttinen, "Design and Implementation of a Portable Long-Term Physiological Signal Recorder," IEEE T. Inf. Technol. Biomed., vol. 14, no. 3, pp. 718-725, May 2010

[5] Pascal Laferriere, Adrian D. C. Chan, and Edward D. Lemaire, "Surface Electromyographic Signals using a Dry Electrode," in MeMeA '10, IEEE International Workshop on, Ottawa, 2010, pp. 77-80.

[6] Silvester Fuhrhop and Stefan Lamparth, "A Textile Integrated LongTerm ECG Monitor with Capacitively Coupled Electrodes," in BioCAS 2009, IEEE, Beijing, 2009, pp. 21-24.

[7] Merja M. Puurtinen, Satu M. Komulainen, Pasi K. Kauppinen, and Jaakko A. V. Malmivuo, "Measurement of noise and impedance of dry and wet textile electrodes, and textile electrodes with hydrogel," in EMBS '06. 28th Annual International Conference of the IEEE, New York City, 2006, pp. 6012-6015.

[8] Roger Abächerli et al., "Performance study of textile electrocardiogram 
electrodes based on heart rate variability," in ISCE 32nd Annual Conference, 2007, p. 30

[9] Jasbir Sra et al., "Posterior left atrial-esophageal relationship throughout the cardiac cycle," J. Interv. Card. Electrophysiol., vol. 16, pp. 73-80, November 2006.

[10] Mariano Noel Benzadón et al., "Comparison of the Amplitude of the PWave from Intracardiac Electrocardiogram Obtained by Means of a Central Venous Catheter Filled With Saline Solution to That Obtained Via Esophageal Electrocardiogram," Am. J. Cardiol., vol. 98, no. 7, pp. 978-981, October 2006

[11] Robert Arzbacher, Janice Jenkins, Steve Collins, and Susan Lorenzen, "Feasibility of Long-Term Esophageal Electrocardiography in the Study of Transient Arrhythmias," Biomed. Sci. Instrum., vol. 14, pp. 1-6, April 1978.

[12] Janice Jenkins, Delon Wu, and Robert Arzbaecher, "Computer diagnosis of supraventricular and ventricular arrhythmias. A new esophageal technique," Circulation, vol. 60, pp. 977-987, 1979.

[13] J. Ramos, M. Vargas, M. Fernández, J. Rosell, and R. Pallás-Areny, "A System for Monitoring Pill Electrode Motion in Esophageal ECG," in EMBS 1993. 15th Annual International Conference of the IEEE, San Diego, 1993, pp. 810-811.

[14] John G. Webster, "Reducing Motion Artifacts and Interference in Biopotential Recording," IEEE Trans. Biomed. Eng., vol. BME-31, no. 12, pp. 823-826, December 1984.
[15] Jörg Ottenbacher et al., "Reliable Motion Artifact Detection for ECG Monitoring Systems with Dry Electrodes," in EMBS '08. 30th Annual International Conference of the IEEE, Vancouver, 2008, pp. 1695-1698.

[16] Steen Pehrson et al., "The Optimal Oesophageal Pacing Technique," Scand. Cardiovasc. J., vol. 33, pp. 103-109, June 1999.

[17] Leif Sörnmo and Pablo Laguna, Bioelectrical Signal Processing In Cardiac and Neurological Applications, 1st ed. Lund, Sweden: Elsevier Inc., 2005.

[18] IEC 60601-2-47, Medical electrical equipment-part 2-47: particular requirements for the safety, including essential performance, 2001.

[19] Monson H. Hayes, Statistical Digital Signal Processing And Modeling, 1st ed. Georgia, USA: John Wiley \& Sons, Inc., 1996.

[20] L. A. Geddes and R. Roeder, "Criteria for the Selection of Materials for Implanted Electrodes," Ann. Biomed. Eng., vol. 31, no. 7, pp. 879-890, July 2003.

[21] Javier Rosell, Josep Colominas, Pere Riu, Ramon Pallas-Areny, and John G. Webster, "Skin Impedance From $1 \mathrm{~Hz}$ to $1 \mathrm{MHz}$," IEEE. Trans. Biomed. Eng., vol. 35, no. 8, pp. 649-651, August 1988. 